\title{
Current methods of assessing blood glucose control in diabetes
}

\author{
MARK EVANS
}

\begin{abstract}
The $\mathrm{HbA}_{1 \mathrm{c}}$ measurement effectively "averages" blood glucose over the previous few months, but can conceal wide variations during that period and patients with apparently well-controlled $\mathrm{HbA}_{1 c}$ may have very different risks of potentially debilitating hypoglycaemia. Self-monitoring of blood glucose provides only "snapshots" of glycaemic control that are unlikely to reveal the true extent of glucose variability. Continuous glucose monitoring (CGM) systems offer a far more comprehensive 24-hour picture of glycaemia, but need regular calibration, are expensive, and limited by short sensor life. Also, the sheer volume of data from CGM downloads can render it difficult to identify patterns. The ambulatory glucose profile (AGP) presents glucose data in a standardised manner, with glucose values presented as a median with 10th, 25th, 75th and 90th centiles. Examination of the AGP can reveal patterns highlighting areas of hypoglycaemia risk or excessive glucose variability, in a way that helps patients to understand and accept the needed changes to their treatment. Software for analysis is available for current systems to indicate possible areas for attention, for example a simple traffic light system where the colour red shows a possible area of risk. AGP could make the difference between identifying or missing a glycaemic pattern and an associated effective therapeutic change, within the timepressured and resource-limited healthcare environment. Finally, the newer technique of "flash glucose monitoring" involves a small factory calibrated sensor which can store glucose data for up to $\mathbf{8}$ hours; users use a handheld receiver to "interrogate" the sensor rather than having information pushed through continuously as in CGM.

Br J Diabetes 2016;16(Suppl1):S7-S9
\end{abstract}

Key words: $\mathrm{HbA}_{1 \mathrm{c}}$, flash glucose monitoring, continuous glucose monitoring, hypoglycaemia, ambulatory glucose profile

Wellcome Trust/ MRC Institute of Metabolic Science, Institute of Metabolic Science Metabolic Research Laboratories, University of Cambridge, UK

Address for correspondence: Mark Evans

Wellcome Trust/ MRC Institute of Metabolic Science, Institute of

Metabolic Science Metabolic Research Laboratories, University of

Cambridge, Robinson Way, Cambridge, CB2 0QQ, UK

E-mail: mle24@cam.ac.uk

http://dx.doi.org/10.15277/bjd.2016.071
Measurements of glycaemic control

$\mathrm{HbA}_{1 \mathrm{c}}$ measurement

Measurement of $\mathrm{HbA}_{1 \mathrm{c}}$ is probably the most widespread method for assessing blood glucose control and has clear, significant merit as a standardised method with an established association with the risk of microvascular complications. The major drawback of $\mathrm{HbA}_{1 c}$ as a single metric is that it gives no information about glycaemic variability. For example, it is quite possible for a patient to have apparently excellent glycaemic control with an $\mathrm{HbA}_{1 c}$ in target values, yet with their daily life being blighted by regular debilitating hypoglycaemia. There are also cases where $\mathrm{HbA}_{1 c}$ is clearly disparate from other measures of glycaemia, either because of known changes in red blood cell physiology (e.g. haemoglobinopathies), suspected inaccuracy (e.g. high $\mathrm{HbA}_{1 c}$ with normal self-monitored blood glucose [SMBG], raising concerns about veracity of SMBG) or other suspected but unproven biological differences (e.g. those who have tissue proteins more or less likely to be glycated).

Self-monitored blood glucose

$\mathrm{HbA}_{1 c}$ can be supplemented by SMBG, captured either in paper diaries or logs, mobile phone apps or simply by viewing and downloading glucose meters brought to clinic. Again there are limitations; any who have tried to maintain records, even over a short period of time, will be sympathetic with the discipline required to perform, let alone sustain, this activity. Records, where kept, may be patchy, inaccurate and/or may not be brought to clinical reviews. Blood glucose meters may also not be available at review appointments or may not be easily downloadable. Where SMBG data are available, the record only gives snapshots of information about discrete time points at which capillary glucose tests are performed.

\section{Continuous glucose monitoring}

Continuous glucose monitoring (CGM) is relatively infrequently used in the UK but has the potential to offer a far more comprehensive 24-hour picture of glycaemia. Most currently available systems are self-inserted and provide on-going real time data transmitted wirelessly to a handheld receiver, or sometimes to a receiver within an insulin pump. Alarms in receivers can be set for low or high values, or to warn of rapidly changing glucose levels. The Medtronic iPro system is "blinded" with data being downloaded after wearing for retrospective pattern analysis by healthcare professionals. The challenges with current CGM systems are that (1) they need calibration against SMBG, thus creating potential for operator error; (2) they are relatively expensive 
Figure 1. Examples of glucose monitoring systems

a) The FreeStyle Libre system

(flash glucose monitoring) b) FreeStyle Navigator II system (continuous glucose monitoring) c) Self-monitoring of blood glucose
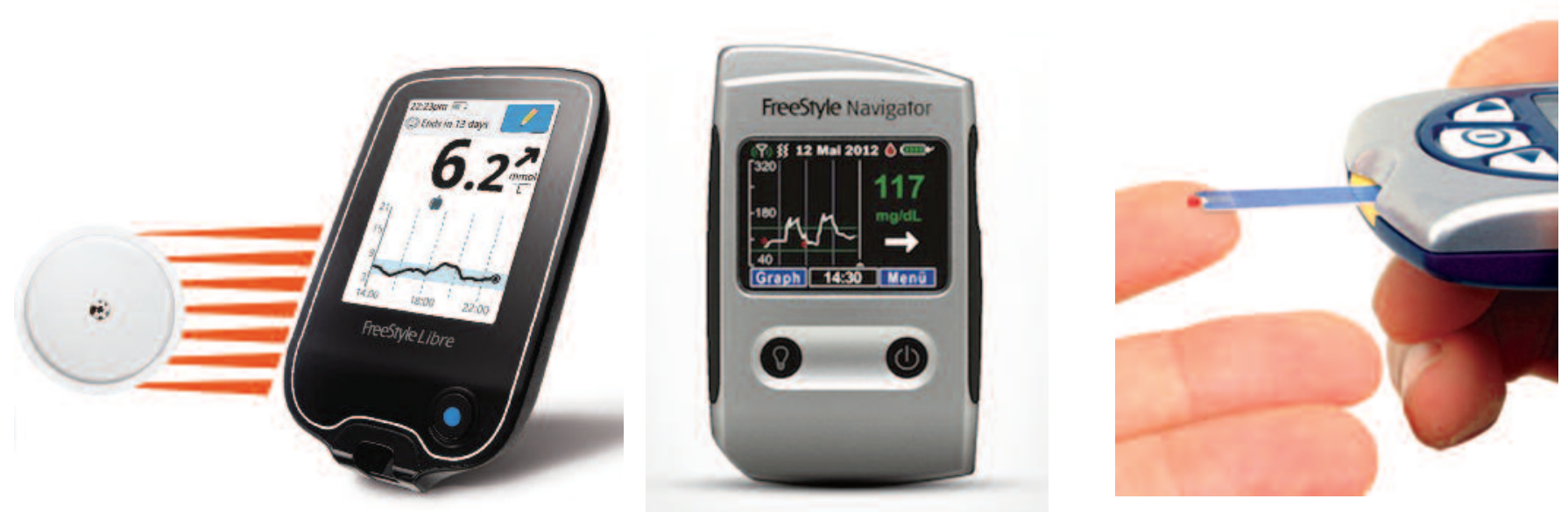

and thus establishing cost effectiveness has been challenging; and (3) systems only last for up to a week at most. There is a temptation to try to extend the period of use off label and against manufacturers' advice and known performance and safety data. Even a cursory internet search will show a number of blogs in which patients describe "hacking" their CGM systems to achieve this.

\section{Flash glucose monitoring}

"Flash" glucose monitoring (GM) has recently emerged as a technology occupying a clinical space between CGM and SMBG. In flash GM, interstitial glucose is measured using the FreeStyle Libre system (Figure 1a) with glucose data being stored for up to 8 hours on the FreeStyle Libre sensor and users use a handheld FreeStyle Libre reader to "interrogate" the sensor rather than information being pushed through as in CGM. Other key differences are that the FreeStyle Libre sensor is factory-calibrated rather than needing calibration against SMBG, and the sensors can be worn for up to 14 days (e.g. Figure 1a).

\section{Current methods for interpreting blood glucose data in diabetes}

"Here and now" measurement and retrospective analysis of blood glucose data

Patients and/or their clinicians need to analyse and act on glucose data, irrespective of the capture method used. The simplest strategy for this is the "here and now" measure, i.e. someone wants to know what their blood glucose is at a particular time, perhaps to check that they are not out of range (e.g. hypoglycaemic) and/or to use that information to determine appropriate insulin dosing before a meal. Less frequently, some may also test diligently and regularly, recording information, but without the data being used to alter management in any tangible way.

"Here and now" glycaemic data may be analysed retrospectively by patients and their clinical teams to look for patterns of glycaemia. Ideally, this leads to appropriate changes in therapy (e.g. insulin doses) or may reveal the impact on blood glucose of certain activities (e.g. of a particular food or exercise regimen), again hopefully leading to an appropriate change in management.

Analysing glucose data retrospectively is challenging and probably done infrequently in real world practice. To aid this, paper diaries are typically organised so that a similar time of day can be viewed in columns. Electronic downloads from meters vary and some merely give a chronological plot of glucose values which challenges interpretation. Others, simple spreadsheets or systems such as Diasend, can colour code out-of-range measurements to help draw the eye. This can be onerous enough with SMBG but with CGM and/or flash GM, the sheer volume of data captured means that simply eyeballing the measures is time consuming and likely to miss important patterns. When CGM was first available, data were typically presented as modal days, which could then be superimposed analogous to viewing columns of data in a paper diary. In practice, this can very quickly come to resemble a plate of spaghetti with major challenges in picking out patterns (Figure 2).

The ability to interpret glucose results is critical to harnessing the power of technology, i.e. the data should result in a therapeutic change rather than being collected for posterity. The presentation of glucose data needs to be in a format that works for patients and non-expert clinicians, not only for expert interpretation. Even better, the presentation could be combined with some algorithm-driven preliminary analysis to indicate possible areas for attention.

\section{Ambulatory glucose profile}

The ambulatory glucose profile (AGP) was developed by Roger Mazze and colleagues. ${ }^{1}$ It is disarmingly simple at face value and aims to present glucose data in a standardised manner analogous to the presentation of cardiac electrical activity in a stan- 
Figure 2. Overlaid continuous glucose monitoring data from several days in the same patient

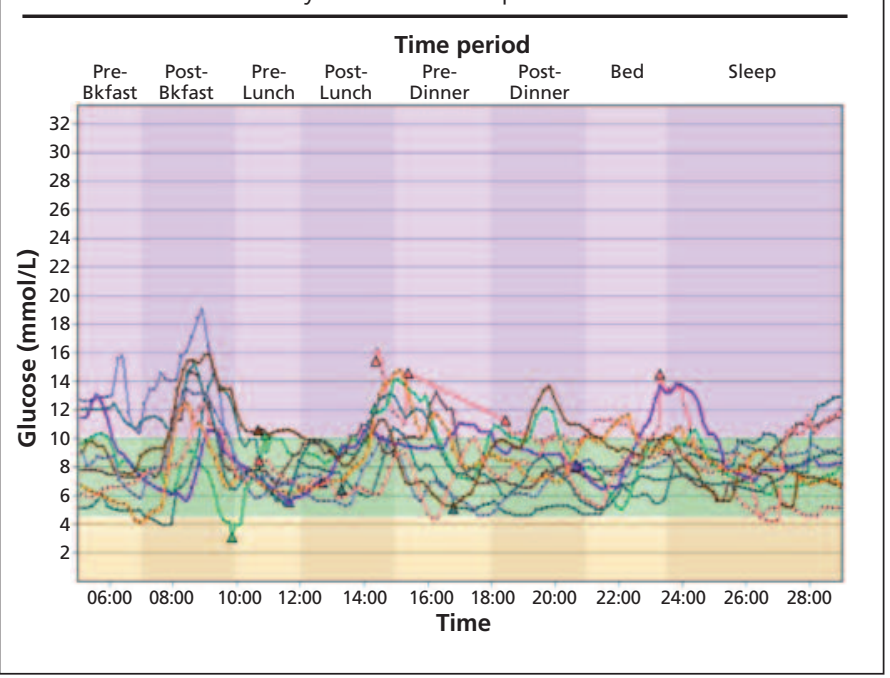

dardised ECG. Glucose values are presented as a median with 10th, 25th, 75th and 90th centile lines (Figure 3).

The data can be scanned rapidly to see whether readings are within the target range and how this varies over a 24-hour period, looking for particular times of hypoglycaemia risk and also for variability from the width of the interquartile range. The AGP thus provides quantitative 24-hour data in a format that facilitates visual recognition of patterns that is comprehensible to a non-expert. This is important as the rationale for suggested alterations in therapy needs to be appreciated by patients, carers and others required to "buy in" to changes. For patients, a simplified AGP is presented on the screen of the handheld FreeStyle Libre reader with the full version viewable when data are downloaded to a home PC or Mac using the FreeStyle Libre software.

The AGP has also been adapted to incorporate some analysis to indicate possible areas for attention, presented as a simple traffic light system where the colour red shows a possible risk. Currently, the AGP can be generated from either the FreeStyle Libre system, or from the FreeStyle Navigator II (Figure 1). At the time of writing, the launch of the FreeStyle Libre system has been globally popular and oversubscribed. Accordingly, most early

Figure 3. Example of an ambulatory glucose profile

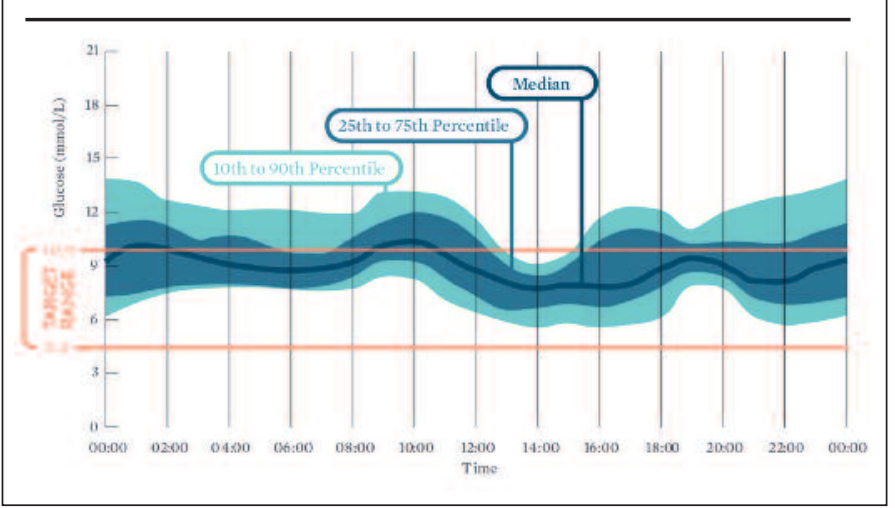

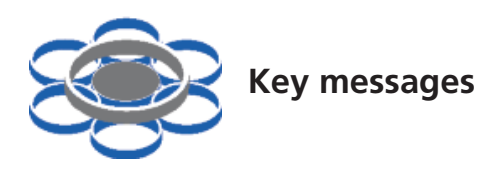

- Continuous glucose monitoring (CGM) is an advance over fingerstick monitoring of blood glucose, but is limited by expense, short sensor life and difficulty in interpreting meter downloads

- The newer technique of "flash glucose monitoring" involves glucose data being stored for up to 8 hours on the sensor; users use a handheld receiver to "interrogate" the sensor rather than having information pushed through continuously as in CGM

- The ambulatory glucose profile provides a means of converting dense glucose data from multiple days into a visual format that facilitates identification of periods of risk of hypoglycaemia or hyperglycaemia

uptake of flash GM in the UK has been self-funded so that clinical teams may not always be aware that their patients have started to use the FreeStyle Libre system. There is therefore a growing likelihood that patients may present to their healthcare professionals with a device and/or AGP download which they are unfamiliar with! Other CGM and glucose monitoring device companies may start to incorporate AGP into their reporting systems, making this facility yet more widespread.

Finally, what evidence is there that AGP offers improved and more accurate interpretation of glucose data? Currently this is limited to an expert panel recommendation for AGP as an effective standard for analysing glucose data. ${ }^{2-4}$ Ideally, the technology now needs studying to determine benefit in terms of time and efficiency for health care professionals and users themselves in interpreting complex glycaemic data sets. Time is more than money here; in time-pressured and resource-limited healthcare systems, this may make the difference between identifying or missing a glycaemic pattern and an associated effective therapeutic change.

Conflict of interest MLE has received speakers and writing fees and/or honoraria from Abbott Diabetes Care, Medtronic, Roche, NovoNordisk, Eli Lilly and CellNovo

Funding This supplement has been supported by a grant from Abbott Diabetes

\section{References}

1. Mazze RS, Lucido D, Langer O, Hartmann K, Rodbard D. Ambulatory Glucose Profile: representation of verified self-monitored blood glucose data. Diabetes Care 1987;10:111-17. http://dx.doi.org/10.2337/diacare.10.1.111

2. Bergenstal RM, Ahmann AJ, Bailey T, et al. Recommendations for Standardizing Glucose Reporting and Analysis to Optimize Clinical Decision Making in Diabetes: The Ambulatory Glucose Profile. J Diabetes Sci Technol 2013;7:562-78. http://dx.doi.org/10.1177/193229681300700234

3. Matthaei $\mathrm{S}$. Assessing the value of the Ambulatory Glucose Profile in clinical practice. $\mathrm{Br} J$ Diabetes Vasc Dis 2014;14:148-52. http://dx.doi.org/10.15277/bjdvd.2014.045

4. Matthaei S, Dealaiz RA, Bose E, et al. Consensus recommendations for the use of Ambulatory Glucose Profile in clinical practice. $\mathrm{Br} J$ Diabetes Vasc Dis 2014;14:153-7. http://dx.doi.org/10.15277/bjdvd.2014.046 